\title{
ESTAMOS VIVENDO UMA EPIDEMIA DE ALERGIA ALIMENTAR?
}

\section{Are we experiencing a food allergy epidemic?}

\section{Fabiane Pomiecinski}

Universidade de Fortaleza - UNIFOR - Fortaleza (CE) - Brasil

\section{Virginia Maria Costa de Oliveira Guerra}

Universidade de Fortaleza - UNIFOR - Fortaleza (CE) - Brasil

\section{Rita Erotildes Maranhão Mariano}

Secretaria da Saúde de Fortaleza - Fortaleza (CE) - Brasil

\section{Raquel Cristina de Sousa Lima Landim}

Universidade de Fortaleza - UNIFOR - Fortaleza (CE) - Brasil

A alergia alimentar é caracterizada por uma reação adversa à ingestão de alimentos, ou aditivos alimentares, mediada por mecanismos imunológicos ${ }^{(1)}$. Os alimentos mais comumente envolvidos nesse processo são: leite de vaca, ovo, trigo, soja, frutos do mar, peixes, amendoim e castanhas. Mas tem ocorrido também por alimentos antes não identificados como causadores de alergias, como kiwi, gergelim, macaxeira (aipim), entre outros ${ }^{(2)}$.

Pressupõe-se que as reações alérgicas aos alimentos acometam cerca de 6 a $8 \%$ das crianças com menos de 3 anos de idade e 2 a $3 \%$ dos adultos, cuja sintomatologia tem se tornado mais grave e mais persistente ${ }^{(2)}$.

As crianças representam o segmento mais susceptível a essas manifestações. E, embora não haja dados oficiais sobre a incidência de alergia alimentar no Brasil, estudos observacionais e relatos dos pediatras gastroenterologistas afirmam se tratar de um problema nutricional em ascensão, que vem se tornando um problema de saúde pública em todo o mundo, causando impacto negativo na qualidade de vida da população.

Vários motivos podem contribuir para esse aumento, principalmente os fatores genéticos e ambientais. Estima-se que o risco de alguém se tornar alérgico seja $60 \%$ definido pela genética e $40 \%$ definido pelo ambiente e hábitos de vida ${ }^{(1)}$.

Quanto à genética, geralmente o indivíduo com alergia alimentar nasce com uma predisposição para desenvolvê-la. Na presença de atopia familiar, é comum os pais ou irmãos apresentarem rinite, asma, dermatite atópica ou alergia alimentar ${ }^{(3,4)}$.

Dentre os fatores ambientais, destacam-se as alterações na microbiota intestinal (uso excessivo de antibióticos, medicamentos inibidores de ácido gástrico, aumento dos nascimentos por cesariana); exposição a alimentos processados, ultraprocessados e transgênicos; baixos índices de aleitamento materno e oferta tardia dos alimentos sólidos às crianças ${ }^{(5)}$.

Infere-se, no entanto, que os maiores desafios diante de uma possível epidemia de alergia alimentar na atualidade estejam relacionados ao tipo de parto, à ausência de amamentação e à exposição a antígenos.

Partindo-se da constatação de que as crianças nascem livres de germes e que a colonização é iniciada imediatamente após o nascimento, compreende-se que esse processo é influenciado pelo tipo de parto. No parto normal, a colonização do recémnascido tem como fonte natural a microbiota intestinal materna, que o "contamina" beneficamente, o que não ocorre durante a cirurgia cesariana. Portanto, o parto normal apresenta vantagens na prevenção dos riscos de alergias, doenças autoimunes e doença celíaca e inflamatória intestinal ${ }^{(6,7)}$.

Outro fator preventivo importante é a prática do aleitamento materno exclusivo. Os principais componentes do leite humano que agem como fator de proteção contra infecções e alergias são: linfócitos (células T de memória) e macrófagos, imunoglobulina do leite humano IgA polimérica, a composição de ácidos graxos poliinsaturados e poliaminas, além de fatores imunomoduladores e anti-inflamatórios (lactoferrina, anticorpos IgM, IgG e IgA, neutrófilos, citocinas) e fatores de crescimento, que provavelmente protegem contra sensibilização alérgica durante o período de aleitamento e pouco depois do fim do aleitamento(8).

Os órgãos de saúde, nacionais e internacionais, recomendam o aleitamento materno exclusivo até os seis meses de idade e após esse período, até os dois anos, complementando a alimentação com alimentos sólidos ${ }^{(9)}$. Apesar de não estar definitivamente comprovado que o leite humano previne a sensibilização a alérgenos, nem que a dieta da mãe durante a gravidez influencia o 
desenvolvimento de alergia alimentar, por todas as suas vantagens nutricionais, imunológicas, econômicas e psicológicas, o aleitamento materno exclusivo deve ser incentivado ${ }^{(10)}$.

A dieta ofertada à criança representa outro fator de prevenção, pois dependendo da sua composição e época do início da alimentação complementar, pode ter vasta influência na composição da microbiota intestinal da criança, a qual pode contribuir para o aparecimento e duração da alergia.

Portanto, os alimentos devem ser incluídos lentamente, um a um, não antes dos 4 meses de idade e sem que seja interrompido o aleitamento materno. A introdução deve acontecer entre os 4 e 6 meses de vida, período chamado de "janela imunológica", pois fora desse período as chances de desenvolver alergias alimentares são maiores ${ }^{(10)}$.

A exclusão de potenciais alérgenos da dieta não é recomendada, pois a exposição precoce a um alimento pode ter um efeito protetor, logo, não se deve retardar o início da oferta dos alimentos sólidos às crianças. Para os já comprovadamente alérgicos, o contato com quantidades muito pequenas do alérgeno, de forma controlada, por via oral, pode ajudar na aquisição de tolerância, sendo esse método conhecido como tratamento de dessensibilização ou imunoterapia oral ${ }^{(3)}$.

Alguns alimentos podem ajudar na adaptação da criança à alimentação complementar. A dieta com fibras pode ser uma aliada na prevenção das alergias por influenciar a diversificação da microbiota. Existem também fórmulas infantis adicionadas de prebióticos que apresentam efeitos positivos, no caso de crianças que não podem ser amamentadas ${ }^{(7,11,12)}$.

Entretanto, apesar da alergia alimentar ser estudada há muitas décadas, ainda são poucos os conhecimentos acerca dos fatores de risco, patogenia, processos celulares e moleculares. Observa-se, contudo, que as fórmulas infantis utilizadas na alergia alimentar estão se tornando mais eficazes e acessíveis ao uso domiciliar e hospitalar, melhorando a qualidade de vida dos portadores de alergia alimentar, mas devem ser utilizadas com parcimônia e somente prescritas dentro dos protocolos estabelecidos pelas sociedades cientificas.

Dentro desse cenário atual, as ações de promoção da saúde e prevenção das alergias alimentares devem ser estimuladas, mediante utilização das diretrizes dos Guias Alimentares na capacitação dos profissionais de saúde, na orientação aos usuários do Sistema Único de Saúde, no atendimento às crianças menores de 2 anos, na Atenção Primária e nos consultórios de puericultura, para fortalecer a implementação da Estratégia Nacional para Promoção da Alimentação Saudável (ENPACS) ${ }^{(9)}$.

O Guia para População Brasileira é outro instrumento que traz um conjunto de conceitos, princípios, diretrizes e estratégias que objetivam proporcionar aos indivíduos e profissionais a promoção de práticas alimentares apropriadas no âmbito individual e coletivo, a fim de promover a saúde e segurança alimentar e nutricional da população ${ }^{(13)}$.

Em suma, não há dúvidas de que a prevenção é o melhor caminho no contexto de provável epidemia de alergia alimentar, cabendo aos profissionais que atendem a clientela materno infantil utilizarem ações de prevenção primária, secundária e terciária ${ }^{(14)}$.

\section{REFERÊNCIAS}

1. Chapman JA, Bernstein IL, Lee RE, Oppenheimer J. Food allergy: a practice parameter. Ann Allergy Asthma Immunol. 2006 Mar;96(3 Suppl 2):S1-68.

2. Nowakg-Wergrzyn A, Sampson HA. Adverse reactions to food. Med Clin North Am. 2006;90(1):97-127.

3. Fiocchi A, Brozek J, Schünemann H, Bahna SL, von Berg A, Beyer K, et al. World Allergy Organization (WAO) Diagnosis and Rationale for Action against Cow's Milk Allergy (DRACMA) Guidelines. Pediatr Allergy Immunol. 2010;21 Suppl 21:1-125.

4. Pereira ACDS, Moura SM, Constant PBL. Alergia alimentar: sistema imunológico e principais alimentos envolvidos. Semina Ciênc Biol Saúde. 2008;29(2):189-200.

5. Luyt D, Ball H, Makwana N, Green K, Brasvin S, Nasser SM, et al. BSACI guideline for the diagnosis and management of cow's milk allergy. Clin Exp Allergy. 2014;44(5):642-72.

6. Pomiescinski F, Yang AC, Navarro-Rodrigues T, Kalil J, Castro FFM. Sensitization to foods in gastroesophageal reflux disease and its relation to eosinophils in the esophagus: is it of clinical importance? Ann Allergy Asthma Immunol. 2010;105(5):359-63.

7. Penders J, Thijs C, Vink C, Stelma FF, Snijders B, Kummeling I, et al. Factors influencing the composition of the intestinal microbiota in early infancy. Pediatrics. 2006;118(2):511-21.

8. Neu J, Rushing J. Cesarean versus vaginal delivery: Long-term infant outcomes and the hygiene hypothesis. Clin Perinatol. 2011; 38(2):321-31.

9. Ministério da Saúde (BR), Secretaria de Atenção à Saúde, Departamento de Atenção Básica. Dez passos para uma alimentação saudável: guia alimentar para crianças menores de dois anos : um guia para o profissional da saúde na atenção 


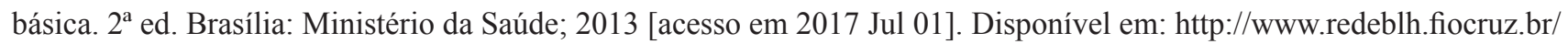
media/10palimsa_guia13.pdf

10. Cocco RR, Souza FS, Sarni RO, Mallozi MC, Solé D. O papel da nutrição no primeiro ano de vida sobre a prevenção de doenças alérgicas. Rev Bras Alerg Imunopatol. 2009;32(2):68-71.

11. Philippi St, organizadora. Pirâmide dos alimentos: fundamentos básicos da nutrição. $2^{\mathrm{a}}$ ed. Barueri: Manole; 2014.

12. Sierra C, Bernal MJ, Blasco J, Martínez R, Dalmau J, Ortuño I, et al. Prebiotic effect during the first year of life in healthy infants fed formula containing gos as the only prebiotic: a multicentre, randomised, double-blind and placebo-controlled trial. Eur J Nutr. 2015;54(1):89-99.

13. Ministério da Saúde (BR), Secretaria de Atenção à Saúde, Departamento de Atenção Básica. Guia alimentar para a população brasileira. $2^{\mathrm{a}}$ ed. Brasília: Ministério da Saúde; 2014.

14. Ferreira CT, Seidman E. Food allergy: a practical update from the gastroenterological viewpoint. J Pediatr (Rio J). 2007;83(1):7-20.

\section{Endereço do primeiro autor:}

Fabiane Pomiecinski

Universidade de Fortaleza - UNIFOR

Av. Washington Soares, 1321

Bairro Edson Queiroz

CEP: 60.811-905 - Fortaleza - Ceará

E-mail: fabiane2212@yahoo.com.br

\section{Endereço para correspondência:}

Virginia Maria Costa de Oliveira Guerra

Universidade de Fortaleza - UNIFOR

Av. Washington Soares, 1321/ Bloco H

Bairro Edson Queiroz

CEP: 60.811-905 - Fortaleza - Ceará

E-mail: virginiac@unifor.br 